\title{
Quantum MMIC (QMMIC) VCO's for Wireless Applications
}

\author{
Vijay Nair, Mandar R. Deshpande, Jonathan Lewis, Nada El-Zein, Scott Ageno, Gary Kramer, \\ Marilyn Kyler, Mike Hupp and Herb Goronkin \\ Physical Sciences Research Laboratories \\ Motorola Labs., Motorola Inc., 7700 S. River Parkway, M/D ML34, Tempe, AZ 85284, USA \\ Email: vijay.nair@motorola.com, Phone 480-755-5590, Fax: 480-755-6065
}

\begin{abstract}
The monolithic integration of heterostructure tuneling diodes with other semiconductor devices, such as HFET's, creates novel, quantum functional devices and circuits. The enhanced functionality of these devices enables design of both digital and analog circuits with reduced complexity, smaller size and better performance. Several types of QMMIC VCO's operating in L-band frequency range have been designed and characterized. VCO's achieved output power of 8-10 dBm at L-band frequency range. All VCO's exhibited very low phase noise (in the range of -107 to $-115 \mathrm{dBc} / \mathrm{Hz}$ ) at 1.0 $\mathrm{MHz}$ away from the carrier frequency.
\end{abstract}

\section{INTRODUCTION}

Increasing demand for smaller, faster and better portable communication devices has created tremendous opportunity for innovative design of semiconductor devices, ICs and wireless systems. These applications make severe demands on the device and IC technologies. One new technology to meet such goals is the Quantum-MMIC (QMMIC) technology where the approach is to incorporate tunnel diodes in the circuits [1-5]. These semiconductor devices exhibit a Negative Differential Resistance (NDR) region in their current - voltage characteristics. Many novel circuit configurations that exploit this unique property have been proposed and implemented by integrating tunnel diodes with other semiconductor devices such as HFET's. Several microwave circuits using tunnel diodes have been demonstrated: voltage controlled oscillators (VCO's), power amplifiers, mixers, and smart antennas [6-10].

In this paper we discuss the design and fabrication of various types of QMMIC VCO's. DC and RF characteristics and a comprehensive large signal model of a tunnel diode incorporated in the harmonic balance analysis of circuits are discussed. The design and simulation of different types of VCO's and experimental results of these VCO's are then presented.

\section{AND RF CHARACTERISTICS OF TUNNEL DIODES}

There are many types of tunnel diodes [4,5] such as interband homojunction Esaki type diodes [1], inter-band heterojunction tunnel diodes [5,8] or intra-band resonant tunneling diodes [2, 3]. They have been realized in many different material systems. The basic device characteristics of all of them however are similar. The DC current -voltage characteristics of a Heterostructure Interband Tunnel Diode (HITD) with one quantum well is shown in Fig. 1(a) [8]. A simplified model of a tunnel diode is also shown in Fig. 1(b). In this model $C_{d}$ is the capacitance of the tunnel diode and $\mathrm{R}_{\mathrm{s}}$ is the parasitic series resistance. In this comprehensive DC/RF model [10] a voltage controlled current source (VCCS) is utilized to model the tunneling diode properties. A polynomial fit to the DC current-voltage characteristics is used as the function $f\left(\mathrm{~V}_{\mathrm{a}}-\mathrm{V}_{\mathrm{b}}\right)$. Two port s-parameter measurements were carried to determine RF equivalent circuit parameters. $R_{s}$ and $C_{d}$ are determined from s-parameters measured at one bias in the NDR region. This model predicts both the DC and RF properties of the diode with a high degree of accuracy over the entire bias range (positive as well 
as negative differential resistance regimes) of the diode. The real part of the diode impedance, computed from s- parameters is shown in Fig. 1(c). When the diode is biased in the NDR region $\left(\mathrm{V}_{\mathrm{d}}\right.$ $=0.2 \mathrm{~V}$ and $0.3 \mathrm{~V}$ ), the impedance is negative up to a frequency, $\mathrm{F}_{\max }$. When the diode is biased outside the NDR range $\left(\mathrm{V}_{\mathrm{d}}=0.65 \mathrm{~V}\right.$ case in Fig. $\left.1(\mathrm{c})\right)$ the impedance stays positive over the entire frequency range. The maximum frequency, $F_{\max }$, is defined as the frequency up to which the real part of the input impedance is negative.

\section{QMMIC VCO DESIGN}

Different types of VCO circuits were designed using the comprehensive model discussed above. Harmonic balance analysis using ADS was used to simulate these circuits. One topology of the VCO (Fig. 2(a)) consisted of one tunnel diode, one capacitor and an inductor. In order to improve the output power, another implementation of VCO that incorporates a buffer amplifier was also designed (Fig. 2(b)). In this design the tunnel diode VCO circuit shown in Fig. 2(a) is monolithically coupled with an HFET buffer amplifier through an inductor. Figure 3 shows the ADS Harmonic Balance analysis simulation results of the tuning range and output power of this VCO. A third embodiment of the VCO shown in Fig 2(c) utilized a monolithically integrated tunnel diodeHFET combination (Heterostructure Interband Tunneling FET (HITFET)). A gate-HITFET (tunnel diode monolithically integrated at the gate of the HFET) is utilized for this application. Simulation and experimental results show that the gate-HITFET has higher gain than the FET alone when the tunnel diode of the HITFET is biased in the NDR regime [10]. The diode bias is adjusted to operate in the negative resistance regime. The HFET gate width and the diode area have to be selected properly to optimize the performance of the VCO. The HFET used in the design had a gate width of $200 \mu \mathrm{m}$ and the diode had a total area of $37.5 \mu \mathrm{m}^{2}$. The frequency of oscillation is primarily controlled by the gate voltage and the output power is primarily controlled by the drain voltage. The VCO tuning is accomplished by adjusting the gate and the drain voltages.

\section{QMMIC VCO'S: MEASURED RESULTS}

The output spectrum and phase noise of the VCO's were measured for all three different configurations discussed in the previous section. The tunnel diodes used in the implementation of the circuit designs had a different material structure and width compared to that shown in Fig. 1 and had a wider NDR voltage span. The tunnel diode VCO (Fig. 2a) was operated with the tunnel diode in the NDR region by applying a bias voltage of $-0.64 \mathrm{~V}$. Fig 4(a) shows a photograph of this VCO. The circuit exhibited an oscillation frequency of $1.01 \mathrm{GHz}$ with an output power of $-4 \mathrm{dBm}$. A phase noise of $-115 \mathrm{dBc} / \mathrm{Hz}, 1 \mathrm{MHz}$ away from the center frequency was observed (Fig 5(a)). However the phase noise is very high closer to the center. This is partly attributed to the circuit getting influenced by the external load.

In the second embodiment, a buffer amplifier is monolithically integrated to the tunnel diode VCO (Fig. 2(b)). This VCO, though bigger in size, has higher power output and better phase noise characteristics. This VCO achieved an output power of $4.7 \mathrm{dBm}$, about $8 \mathrm{~dB}$ higher than the VCO that employed only the tunnel diode. The measured output power is not as high as that expected from the simulation (Fig. 3). This is partly due to the differences in the tunnel diode characteristics used for simulation and actual circuit implementation. A phase noise of $-111 \mathrm{dBc} / \mathrm{Hz}$ was also exhibited by this circuit and the phase noise close to center is also much better (figure not shown).

The third configuration of QMMIC VCO circuit (Fig. 2(c), Fig 4(b)) employed a gate-HITFET device. Power output as high as $8.1 \mathrm{dBm}$ has been achieved at the center frequency of $1.01 \mathrm{GHz}$. The VCO exhibited a phase noise $-104 \mathrm{dBc} / \mathrm{Hz}$ at $1.0 \mathrm{MHz}$ away from the center frequency as shown in Fig. 5(b). The performance of this QMMIC VCO is comparable to that for a tunnel diode 
VCO in combination with a buffer amplifier (Fig.2(b)). This circuit however is more compact and has one less inductor since it uses the integrated HITFET device.

\section{CONCLUSIONS}

Different types of QMMIC VCO's using tunnel diodes and integrated tunnel diode-HFET combination are presented. A comprehensive $\mathrm{DC} / \mathrm{RF}$ model that can be incorporated in the Agilent ADS simulation software is illustrated. The circuit design and measured performance of three different types of QMMIC VCO's are discussed. VCO's exhibited output as high as $8 \mathrm{dBm}$ with very low phase noise (in the range of -107 to $-115 \mathrm{dBc}$ ) at $1.0 \mathrm{MHz}$ away from the carrier frequency.

\section{REFERENCES}

[1] Esaki L, "New phenomenon in narrow germanium p-n junctions", Phys. Rev., 109 603, 1958.

[2] Sze S M, "Physics of semiconductor devices, $2^{\text {nd }}$ Edition", John Wiley and Sons, 1981.

[3] Sollner T C L G, Goodhue W D, Tannelwald P E, and Parker C D, "Resonant tunneling through quantum wells at frequencies up to $2.5 \mathrm{THz}$ ", Appl. Phys. Lett., 43, 588, 1983.

[4] Mizutz H and Tanoue T, "The Physics and Applications of Resonant Tunneling Diodes", Cambridge University Press, 1995.

[5] Seabaugh A and Lake R, "Tunnel Diodes”, Encyclopedia of Applied Physics, vol. 22, 335-359, (Wiley), 1997.

[6] V. Nair, N. El-Zein, J. Lewis, M. Deshpande, G. Kramer, M. Kyler, G. Maracas, and H. Goronkin, "X-band Heterostructure Interband Tunneling FET (HITFET) VCOs”, 1998 IEEE GaAs IC conference, pp. 191-193, 1998.

[7] V. Nair, N. El-Zein, G. Kramer, G. Maracas, and H. Goronkin, "RF Applications of Quantum Functional Devices(QFD)”, Progress in Electromagnetic Research Symposium, Nance, France, July 1998.

[8] Deshpande M et al., "Heterojunction Interband Tunneling FETs: Optimization and Use in Amplifier Circuits", Proceedings of $26^{\text {th }}$ Int. Symposium. Compound Semiconductors, 351, 1999.

[9] K. Liu, S.M. El-Ghazaly,V. Nair, N. El-Zein, H. Goronkin, “Active Antennas for Wireless Aplications Using TwoTerminal Quantum Devices," 2000 IEEE Antenna and propagation Society International Symposium, pp. 128083, July 2000.

[10] Deshpande M et al., “A Comprehensive DC/RF Tunnel Diode Model and its Application to Simulate HITFET's (Heterostructure Integrated Tunneling FET's) and Quantum-MMIC's”, Proceedings of $27^{\text {th }}$ Int. Symposium. Compound Semiconductors, Monterey, CA, October 1-5, 2000.

\section{FIGURES}

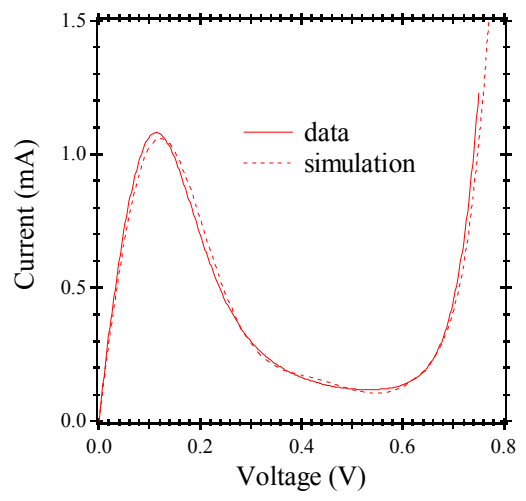

(a)

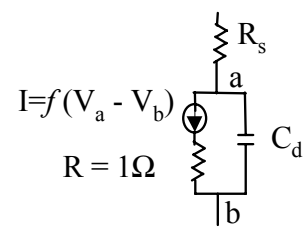

(b)

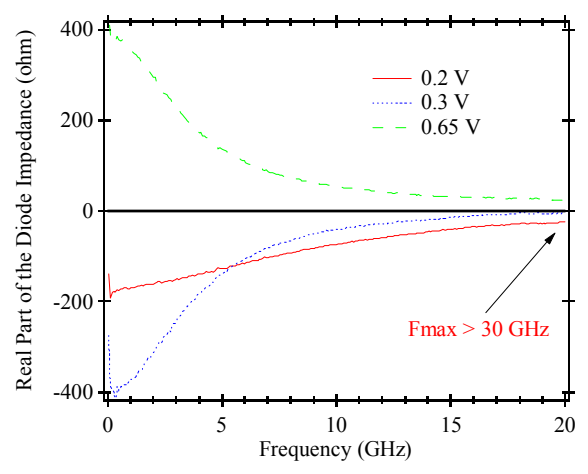

(c)

Fig. 1 (a) DC Current-Voltage characteristics of a tunnel diode showing the negative differential resistance (NDR) region, (b) A comprehensive model of the tunnel diodes and (c) Measured RF characteristics of the tunnel diode at different biases. 


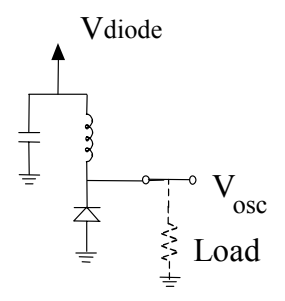

(a)

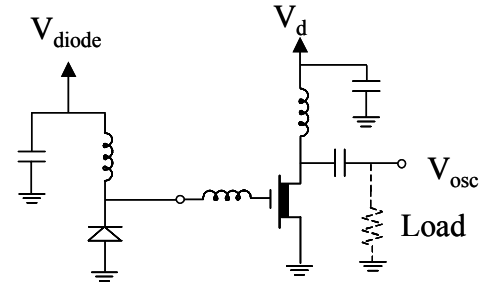

(b)

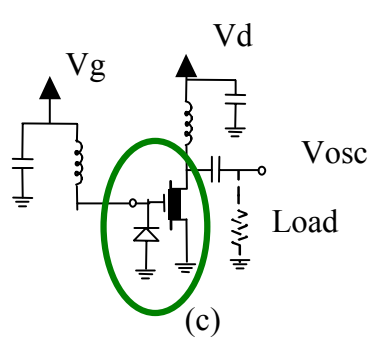

(c)

Fig. 2. Schematics of different VCO's (a) Tunnel Diode VCO, (b) Tunnel Diode VCO with buffer amplifier, and (c) HITFET VCO

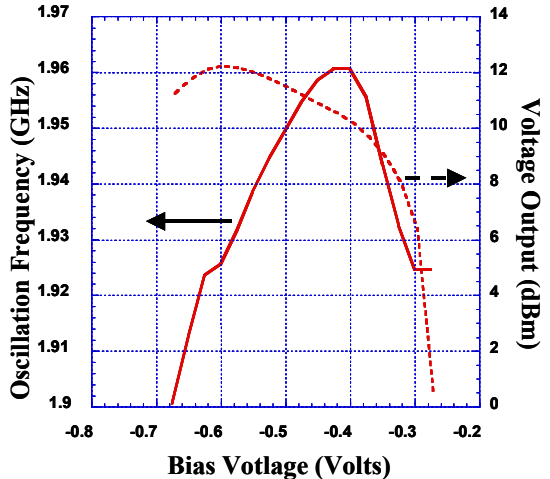

Fig 3. Harmonic balance simulation results for Tunnel Diode VCO with buffer amplifier (Fig. 2(b)).

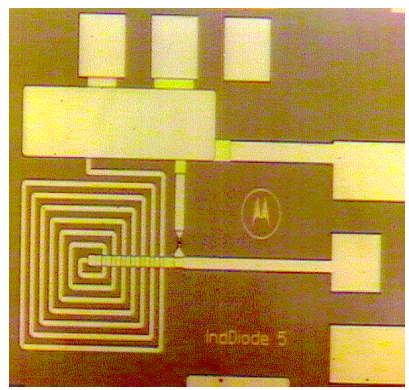

(a)

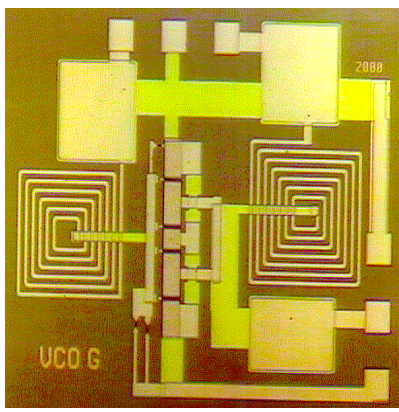

(b)
Fig. 4. Photographs of fabricated circuit of (a) Tunnel Diode VCO and (b) HITFET VCO

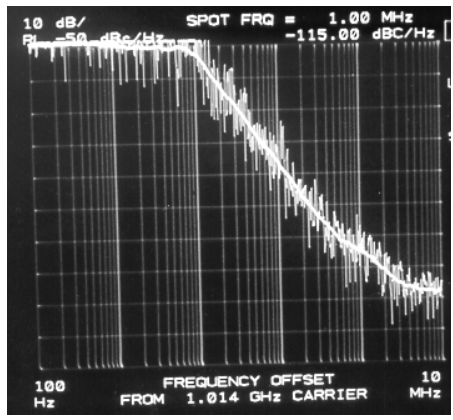

(a)

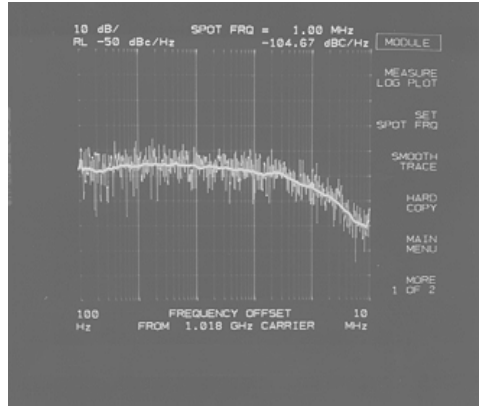

(b)

Fig. 5. Measured phase noise of (a) Tunnel diode VCO (Fig. 2(a)) and (b) HITFET VCO (Fig. 2(c)) 